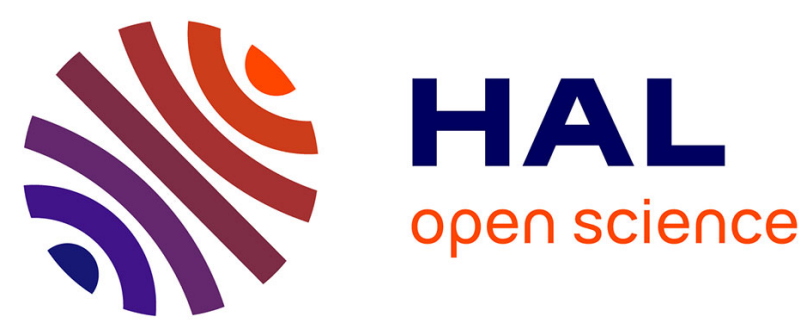

\title{
On the Plastic and Viscoplastic Constitutive Equations - Part I: Rules Developed With Internal Variable Concept
} Jean_louis Chaboche, Gilles Rousselier

\section{To cite this version:}

Jean_louis Chaboche, Gilles Rousselier. On the Plastic and Viscoplastic Constitutive Equations Part I: Rules Developed With Internal Variable Concept. Journal of Pressure Vessel Technology, 1983. hal-03464390

HAL Id: hal-03464390

https://hal-mines-paristech.archives-ouvertes.fr/hal-03464390

Submitted on 1 Jan 2022

HAL is a multi-disciplinary open access archive for the deposit and dissemination of scientific research documents, whether they are published or not. The documents may come from teaching and research institutions in France or abroad, or from public or private research centers.
L'archive ouverte pluridisciplinaire HAL, est destinée au dépôt et à la diffusion de documents scientifiques de niveau recherche, publiés ou non, émanant des établissements d'enseignement et de recherche français ou étrangers, des laboratoires publics ou privés. 


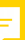

\section{On the Plastic and Viscoplastic Constitutive Equations- Part I: Rules Developed With Internal Variable Concept}

J. L. Chaboche

Section Head,

ONERA,

Châtillton, France

G. Rousselier

Engineer,

EDF, Centre les Renardieres, Moret-sur-Poing, France
The description of monotonic and cyclic behavior of material is possible by generalizing the internal stress concept by means of a set of internal variables. In this paper the classical isotropic and kinematic hardening rules are briefly discussed, using present plastic strain tensor and cumulated plastic strain as hardening variables. Some additional internal variables are then proposed, giving rise to many possibilities. What is called the "nonlinear kinematic hardening" leads to a natural description of the nonlinear plastic behavior under cyclic loading, but is connected to other concepts such as the Mroz's model, limited to only two surfaces, and similarities with other approaches are pointed out in the context of a generalization of this rule to viscoplasticity.
Constitutive equations for the mechanical behavior of materials are generally based on one of the two following thermodynamical concepts [1]:

(i) The present state of the material depends on the present values and the past history of observable variables only (total strain, temperature. . .).

(ii) The present state of the material depends on the present values only of both observable variables and a set of internal state variables.

In this paper, the second approach is considered in order to connect together different theories, showing some similarities, and to propose some additional internal variables giving rise to many possibilities. From the practical point of view, to solve engineering problems by means of the discussed constitutive equations, it is necessary to perform incremental computations of the structure.

In the classical developments of thermodynamics with internal variables, several concepts are introduced, such as:

- the existence of a thermodynamic potential (the free energy for example) from which the relations between state variables (strain, temperature for example) and associated thermodynamic forces (stress, entropy...) are defined;

- the dissipative potential, with generalized normality rule, which gives the evolution equations for internal state variables (plastic strain and others. . .), and allows the a priori verification of the second principle.

In the context of plasticity and viscoplasticity, such general

Contributed by the Pressure Vessels and Piping Division and presented at the Pressure Vessels and Piping Conference, Orlando, Florida, June 27-July 2, 1982, of The American Society of Mechanical Engineers. Manuscrip received at ASME Headquarters, December 9, 1982 concepts have been applied by many workers, giving rise to a coherent tool, especially for the classical flow rules [1-3]. We shall limit the present contribution to the study of differential constitutive equations for the internal variables; the expressiois for corresponding dissipative potentials have been analysed previously [4-5].

One of the basic flow rules is the so-called nonlinear kinematic rule, initially introduced by Armstrong and Frederick [6] and widely developed in the recent few years [7-9]. The use of internal variables playing the role of internal stresses, can be considered as conceptually equivalent to the use of multilayer models as in the theory developed by Besseling [10] and Meijers [11].

The equations discussed in next sections have been applied to several refractory alloys, used in gas turbine applications (IN 100, for example), especially in the high temperature viscoplastic range. The application to the complex cyclic behavior of type 316 stainless steels is considered in the second part of the paper.

\section{Development of a Nonlinear Kinematic Rule}

When dealing with the plastic and/or viscoplastic flow, the central problem concerns the modelization of hardening processes. In terms of a macroscopically homogeneous material, the classical parameters used to describe hardening are:

(i) the cumulated plastic strain $p$, scalar isotropic variable defined by

$$
d p=\left(\frac{2}{3} d \epsilon_{p_{i j}} d \epsilon_{p_{i j}}\right)^{1 / 2}
$$

(ii) the present plastic strain, $\alpha_{i j}=\epsilon_{p i j}$, used as a kinematic variable. 
The classical modelizations introduce the concept of elastic domain (or equipotentials), written in the stress space by means of the thermodynamic forces associated with the two internal variables. Such an elastic domain is

$$
f=J(\sigma-\chi)-R-k \leq 0
$$

where the scalar variable $R$ (or drag stress [12]) is associated with $p$ and the kinematic tensor $\chi$ (or back stress or rest stress tensor [13]) is associated with $\alpha$. They, respectively, give the size and the position of the elastic domain. $J$ represents a distance in the stress space; for a von Mises material

$$
J(\sigma-\chi)=\left[\frac{3}{2}\left(\sigma_{i j}^{\prime}-\chi_{i j}^{\prime}\right)\left(\sigma_{i j}^{\prime}-\chi_{i j}^{\prime}\right)\right]^{1 / 2}
$$

where $\sigma^{\prime}$ and $\chi^{\prime}$ are the deviators of $\sigma$ and $\chi . k$ is a material constant (temperature dependent) corresponding to the initial size of the elastic domain.

The relations between such variables are generally nonlinear for the isotropic variables, but linear for the kinematic ones, which corresponds to the classical rule of Prager [14]

$$
R=\hat{R}(p) \quad \chi=\frac{2}{3} c \alpha=\frac{2}{3} c \epsilon_{p}
$$

The plastic flow follows the normality rule, which states

$$
d \epsilon_{p}=d \lambda \frac{\partial f}{\partial \sigma}
$$

The plastic multiplier $d \lambda$ is derived from the hardening rule through the consistency condition $f=d f=0$ when plastic flow occurs (no plastic flow when $f<0$ or $d f<0$, and $f$ cannot be positive for time independent plasticity).

The usual linear kinematic rule can be easily modified in order to introduce some nonlinear evolution, with an acceptable description of cyclic loadings (concavity of the stressstrain loops under tensile-compressive loading for example). This modification, initially proposed by Armstrong and Frederick [6], in which an evanescent strain memory effect is introduced (evanescent along the plastic strain path), can be written in its simplest form

$$
d \alpha=d \epsilon_{p}-C \alpha d p
$$

or

$$
d \chi=C\left(\frac{2}{3} a d \epsilon_{p}-\chi d p\right) \text { with } C=c / a
$$

Under uniaxial cyclic loading, direct integration gives the following relations for stress in each branch

$$
\begin{gathered}
\sigma=\nu a+\left(\chi_{o}-\nu a\right) e^{-C\left(\epsilon_{p}-\epsilon_{p_{o}}\right)}+\nu R(p)+\nu k \\
=\chi\left(\epsilon_{p}\right) \pm R(p) \pm k
\end{gathered}
$$

where $\nu= \pm 1$, depending on the direction of plastic flow and $\chi_{o}, \epsilon_{p_{o}}$ are the values of $\chi$ and $\epsilon_{p}$ at the last change of plastic strain rate. Let us underline the complete generality of this rule, expressed by equation (5), under any loading condition; the unloading events $\chi_{o}, \epsilon_{p o}$ in (6) appear only after explicit integration. Relation (6) shows also the two different dependencies upon present strain and cumulated plastic strain (the last term allows cyclic hardening or softening).

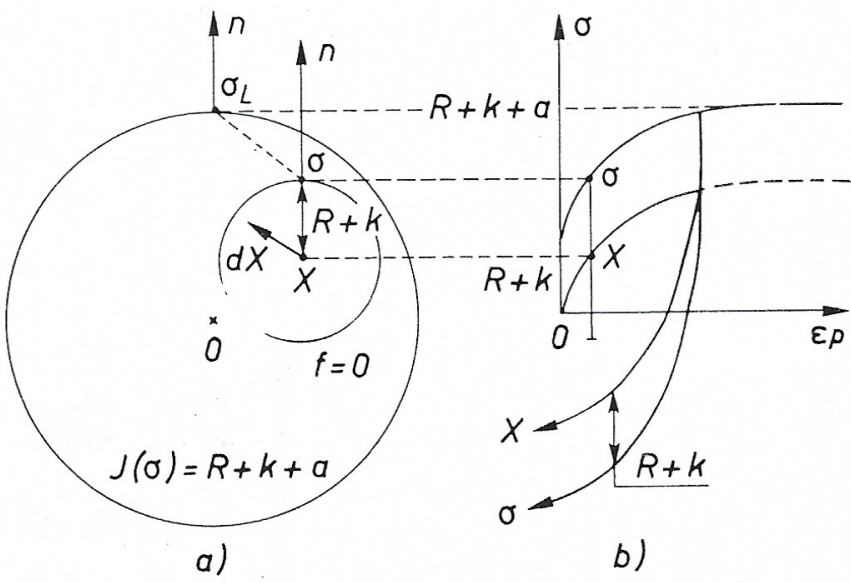

Fig. 1 The nonlinear kinematic rule (for $R+k=C^{t e}$ )-(a) in. terpreted as two-surfaces theory, (b) tensile-compressive response

As shown in Fig. 1(b), the evolution of $\chi$ as a function of $\epsilon_{p}$ is no longer univocal and the concavity of stress-strain loops is correctly represented. Cyclic stabilization implies an asymptotic value for $R(p)$ and the symmetry condition when no ratchetting occurs (obtained from integration) is

$$
\chi_{\operatorname{Max}}=\chi_{o}=a \text { th } C \frac{\Delta \epsilon_{p}}{2}
$$

where $\Delta \epsilon_{p}$ is the plastic strain range at stabilization. Under strain control, the present model leads to a mean stress relaxation effect before stabilization.

Another property of the nonlinear kinematic rule is to give the possibility of ratchetting, when a cyclic load is superimposed to a constant stress state (nonsymmetrical tension-compression or cyclic torsion with constant tension ... .). It is well known that Prager's kinematic rule (or the modified rule by Ziegler [15]) cannot describe such ratcheting effects.

A similar model (as the NLKR) was proposed by Mroz, Shrivastava and Dubey [16], where $\chi$ in equation (5) is replaced by $\epsilon_{p}$. This modification leads to similar results under cyclic loadings but some limitations (in strain) can be necessary.

For the isotropic hardening (equation governing the scalar variable $R$ ) one can use any function $R(p)$, as measured for example from the tension curve (after elimination of the kinematic part). We can also restrict ourselves to some differential equation, similar to (5), leading to a stable asymptotic behavior (which is necessary for cyclic stabilization)

$$
d R=b(Q-R) d p
$$

where $b$ and $Q$ are two constants. The relation between $R$ and $p$ is obtained after integration

$$
R=Q\left(1-e^{-b p}\right)
$$

The foregoing formulation has been generalized by Lee and Zaverl [17], including some slight modifications: they add a fourth-order tensorial variable $M_{i j k l}$ in order to take into

\section{Nomenclature}

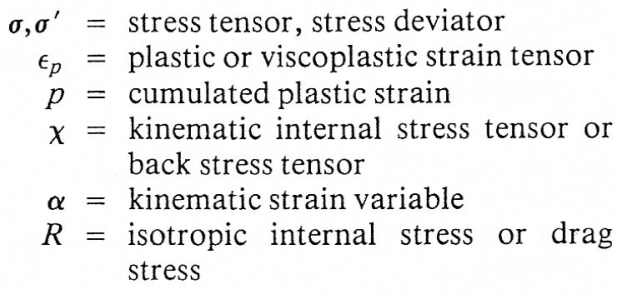

$$
\begin{aligned}
\sigma, \epsilon_{p}, \chi & =\text { tensile components of foregoing } \\
& \text { tensors } \\
J= & \text { Mises-type invariant } \\
G, f, F= & \text { elastic, yield and bounding surfaces } \\
\nu= \pm 1= & \text { tension-compression index } \\
M_{i j k l}= & \text { anisotropic tensor } \\
k, a, C, n, K, b, \gamma, m, w= & \text { material coefficients }
\end{aligned}
$$


account the anisotropy effects, existing the "initial state" or induced by hardening. As proposed in previously by many workers $[18,19,20]$, the von Mises criterion [3] is replaced by

$$
J=\left[M_{i j k l}\left(\sigma_{i j}-\chi_{i j}\right)\left(\sigma_{k l}-\chi_{k l}\right)\right]^{1 / 2}
$$

This allows the simplest kind of anisotropy, described through rotation of the surfaces, which is representative of many experimentally observed second-order effects, especially in stainless steels [21, 22]. A third-order anisotropy with distorsion of the yield surface and appearance of some "corner" effects [23, 24], cannot be described by such a simple criterion.

The original suggestion of Lee and Zaverl was to use $M_{i j k l}$ as an internal state variable, by which evolution could be governed by the same kind of equation

$$
d M_{i j k l}=w\left(M_{i j k l}^{s}-M_{i j k l}\right) d p
$$

where $w$ is a material constant and $M_{i j k l}^{s}$ represents a stationary anisotropic state. The initial state is represented by some tensor $M_{i j k l}^{o}$, for example an isotropic von Mises tensor: $\delta_{i k} \delta_{j l}+\delta_{i l} \delta_{j k}-43 \delta_{i j} \delta_{k l}$. The choice of $M_{i j k l}^{s}$ as a function of the applied loading and the subsequent hardening represents the main difficulty of this approach and has not been clearly defined until now [25].

\section{Relations to Other Approaches}

As mentioned already $[17,26]$, the foregoing internal variable approach of plasticity through a nonlinear kinematic rule can be considered as a two-surfaces theory, as in many other works $[27,28]$. These two surfaces are

1 the yielding surface, which is defined by $f=0$;

2 the bounding surface (or limit surface), which defines the limiting states of stress and can be obtained from the two inequalities: $J(\sigma-\chi) \leq R+k$ and $J(\chi) \leq a$, the last one being easily verified from the rule (5). That gives for the bounding surface

$$
F=J(\sigma)-R-k-a=0
$$

Figure $1(a)$ illustrates this property, also evident from the uniaxial particular case (6). These two surfaces can expand isotropically through the functions $R(p)$ and $a(p)$. The yielding surface moves kinematically by the rule (5), but the bounding one has no kinematic movement; this movement could be superimposed by adding to the $\chi$ variable in (2) a linear kinematic variable (see relations (19) and (20)), giving rise to possibilities similar to that of modelization by Dafalias and Popov $[27,29]$.

A second analogy has to be pointed out: the nonlinear kinematic rule (5) corresponds to a particular Mroz's model [30], with only two surfaces. In the original Mroz's formulation, the present state is defined through a family of surfaces

$$
f_{l}=J\left(\sigma-\chi_{l}\right)-k_{l}=0
$$

$\chi_{l}$ and $k_{l}$ giving, respectively, the position and the size of the surface $f_{l}=0$. The plastic flow, when the stress has attained the surface $f_{l}$, is governed by its movement, until the next surface $f_{l+1}$ is attained. The kinematic movement of the surface is described by the following hypotheses:

(a) the plastic strain rate is defined through the normality rule (4):

$$
d \epsilon_{p}=d \lambda_{l} \frac{\partial f_{l}}{\partial \sigma}=\sqrt{\frac{3}{2}} d \lambda_{l} n
$$

(b) the rate of the kinematic variable $\chi_{l}$ is proportional to $\sigma_{l+1}-\sigma$, where $\sigma_{l+1}$ is the stress state on the next surface $f_{l+1}$ with the same outward normal

$$
d \chi_{l}=d \mu\left(\sigma_{l+1}-\sigma\right)
$$

Such a condition is chosen in order to assure coincidence of normals when actual stress state (on surface $f_{l}$ ) will attain next surface $f_{l+1}$.

In the Mroz's formulation, the multiplier $d \mu$ is determined from the consistency condition $f_{l}=d f_{l}=0$ and the scalar $d \lambda_{l}$ (or the plastic strain increment $d p$ ) can be chosen freely; Mroz used a linear relationship, i.e., a constant value for each $d \lambda_{l}$.

By using the von Mises criterion (3) and the relations (2) and (4), the nonlinear kinematic rule (5) leads to

$$
d \chi=d \chi^{\prime}=C\left[\frac{a}{R+k}\left(\sigma^{\prime}-\chi^{\prime}\right)-\chi^{\prime}\right] d p
$$

If $\sigma_{L}$ is the stress state on the bounding surface (12) corresponding to the same outward normal as the direction of plastic flow, one can write (in the present model the center of this surface is the stress origin, see Fig. 1(a)

$$
\frac{\sigma_{L}^{\prime}}{R+k+a}=\frac{\sigma^{\prime}-\chi^{\prime}}{R+k}
$$

The combination of (16) and (17) easily gives [8]

$$
d \chi=C\left(\sigma_{L}^{\prime}-\sigma^{\prime}\right) d p
$$

which is very similar to the Mroz's hypothesis (15). The only differences are the use of stress deviator in place of stress and of plastic strain increment $d p$ in place of multiplier $d \mu$. The first difference (deviators) does not change anything under the plastic uncompressibility hypothesis as a pure volumetric translation of the yield surface does not change the yield surface.

In fact, the foregoing demonstration implies that the Mroz's model contains the nonlinearity of hardening between two successive surfaces (with the two modifications just mentioned). This remark could lead to a better use of this model, with a few surfaces only for a given nonlinearity in the stress-strain response. Moreover, this is a proof that general properties of the nonlinear kinematic rule (5) and the Mroz's model are identical.

Several experimental studies have shown that the translation of the yield surface follows the direction of prestress (when proportional loading is considered) $[22,23]$. It can be demonstrated that such a behavior is in accordance with most of the kinematic flow rules (Prager [14], Ziegler [15], Mroz [30]) and with the nonlinear kinematic rule developed in the foregoing. Let us remark here that in the principal stress plane $\left(\sigma_{1}, \sigma_{2}, \sigma_{3}=0\right)$, such a translation does not follow the direction of the normal to the von Mises ellipse, even with the Prager's rule, where proportionality between strain increment and translation is supposed. In fact, such a proportionality is written in the deviatoric plane: the corresponding translation of the von Mises cylinder, parallel to the stress deviator (but perpendicular to its axis), accords exactly with a translation of the von Mises ellipse in the plane, following the direction of loading (under proportional loading). This property implies that experimental proportional loadings cannot validate or invalidate the theoretical rules.

The nonlinearity of the rule (5) arises for a limited range in plastic strain, depending on the constant $C$ (Fig. 2(a)). In order to extend its validity to a larger domain in stress and strain one can use several kinematic variables $\chi_{j}$ (two or three), each of them following the same typical rule [31]

$$
d \chi_{j}=C_{j}\left(\frac{2}{3} a_{j} d \epsilon_{p}-\chi_{j} d p\right)
$$

The yield surface is then expressed with the sum of such separated variables

$$
f=J\left(\sigma-\sum_{d} \chi_{j}\right)-R-k=0
$$

As shown in Fig. 2(b) (for a purely kinematic case), such an 
increase in the parameters for the material leads to greater possibilities for the description of stress-strain loops: a large value of $C_{1}$ associated with a small value of $a_{1}$ gives the initial nonlinearity with a very large plastic tangent modulus (but not infinite as in some other approaches), $C_{2}$ and $a_{2}$ give the median nonlinear behavior, a low value of $C_{3}$, associated with a high value of $a_{3}$ describes the asymptotic behavior for large strain, corresponding to the existence of a bounding surface. One can degenerate equation (18) into a linear rule

$$
d \chi_{3}=\frac{2}{3} C_{3} d \epsilon_{p}
$$

Let us mention that the rule (18), (19), is very similar (qualitatively and quantitatively) to the model proposed by Dafalias and Popov, through a different mode of presentation (the kinematic rule is not stated a priori but follows from the evolution of tangent modulus). Two differences have to be pointed out:

(i) In our approach the plastic tangent modulus cannot be infinite at the initiation of yielding as in [29]; (a sufficiently high value is, however, possible with the parameters $a_{1}, C_{1}$ ).

(ii) Under cyclic loading (with or without unloading), the theory developed by Dafalias and Popov needs updating of the tangent modulus, which is not the case with the present formulation. In fact, updating procedures seem to be often introduced to account for the very high nonlinearity in the stress-strain loops (tangent modulus decreasing from infinity to some limit value). The present development shows one possibility to prevent updating with a sufficiently large variation of tangent modulus.

A new approach was recently introduced by Dafalias [32, 33] to eliminate the need for updating: the new concept of "elastic nucleus," avoiding an explicit yielding surface and a load-unload criterion allows a smooth elastic-plastic transition and a better description of flow after partial unloading. Unfortunately, this theory is more difficult to develop with explicit integration procedures and cannot be re-examined in the present context. Future works and evaluations of such attractive concept could certainly improve its understanding and applicability.

\section{Viscoplastic Constitutive Equations}

The viscoplastic flow rules are needed when time or strain rate influence the inelastic behavior. Time-independent plasticity is then considered as a particular limiting case of viscoplasticity. The introduction of viscous effect is easily done, using equidissipative surfaces [13]; a pure elastic domain can still be considered

$$
G=J(\sigma-\chi)-R-k \leq 0
$$

but, as a major difference with the pure elastic case, the stress state can be such as $G>0$. The viscoplastic potential can be expressed as a power function ${ }^{1}$ of $G$, which means that the strain rate will be a power function of $G$. Using a von Mises criterion and the normality rule [7]

$$
d \epsilon_{p}=\frac{3}{2}\left\langle\frac{J(\sigma-\chi)-R-k}{K}\right\rangle^{n} \frac{\sigma^{\prime}-\chi^{\prime}}{J(\sigma-\chi)} d t
$$

The same hardening rules as for plasticity can be used; for example, the nonlinear kinematic hardening defined by equation (5). Some experimental studies have shown the preponderance of such kinematic behavior in the viscoplastic behavior $[23,35]$. The rule (5) was used with success for the IN 100 refractory alloy at several temperatures $[7,9,36]$ and seems to apply also to other metallic materials [35].

Other kinematic rules have been developed in order to

\footnotetext{
${ }^{1}$ A different function can be used as in [12 or 34].
}

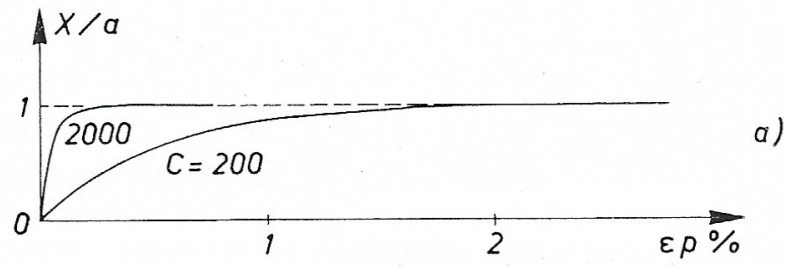

(a) The range of nonlinearity in kinematic hardening depending on coefficient $C$

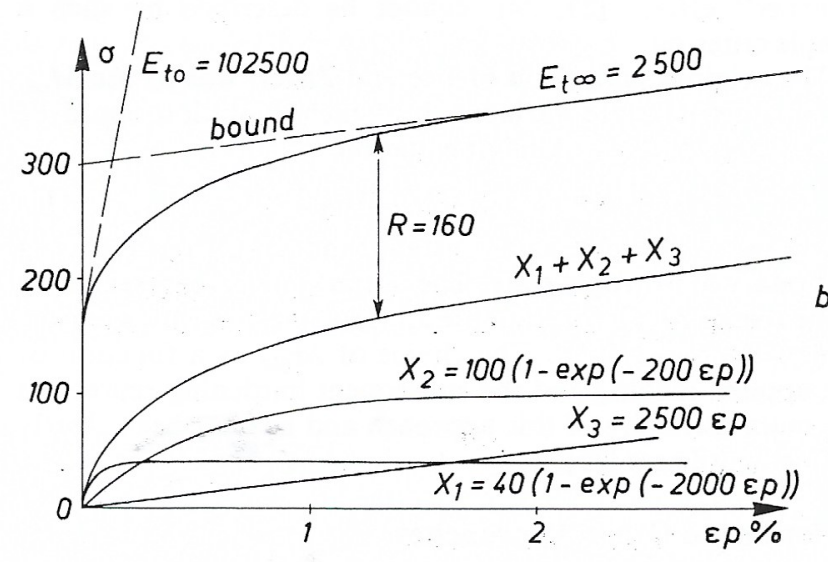

(b) Stress-strain curve obtained from 3 kinematic variables

Fig. 2

describe the viscoplastic flow: the well-known BaileyOrowan equation [37] introduces nonlinear effects through a recovery term

$$
d \chi=C d \epsilon_{p}-\gamma \chi d t
$$

Such a recovery can also be introduced in the isotropic hardening, as in the Lagneborg's model [38] or in MerzerBodner equations [39]. The simple isotropic rule (8) can be generalized by

$$
d R=b(Q-R) d p-\gamma R^{q} d t
$$

The kinematic variable equation (23) leads to nonlinear hardening only by time effect: in other words, for a high strain rate and under cyclic loading (for stabilized behavior one must have $d R=0$ ), the hardening remains linear. Nonlinearity is only present if coefficient $C$ in equation (23) is depending upon stress [40] or back stress $\chi$ [41]. Dependence in terms of invariants of $\sigma$ or $\chi$ gives however unsatisfactory results under cyclic loading.

Superposition of the nonlinear kinematic hardening and nonlinear time recovery is easily obtained by (7).

$$
d \chi=C\left(\frac{2}{3} a d \epsilon_{p}-\chi d p\right)-\gamma J(\chi)^{m-1} \chi d t
$$

which generalizes the Bailey-Orowan equation and is similar to a proposition of Malinin and Khadjinsky. Many history effects can be simulated through such a combination: partial or complete recovery of hardening by aging at high temperature, dependency of the internal stress (or friction stress) on the applied stress and time in the creep tests [42], acceleration effect for creep at a higher stress, creep delay after a decrease in the applied stress [4].

The effect of the evolution of the microstructure upon the macroscopic viscoplastic behavior can be described by means of additional internal variables in the isotropic hardening equation $[5,9]$.

Under uniaxial loading, if we neglect the time-recovery effects $(\gamma<<1)$, the present theory leads to the following stress decomposition

$$
\sigma=\chi\left(\epsilon_{p}\right) \pm R(p) \pm k \pm \sigma_{v}\left(\dot{\epsilon}_{p}\right)
$$

where the kinematic term depends on the last reversal $\epsilon_{p_{\curvearrowleft}}, \chi_{o}$ 


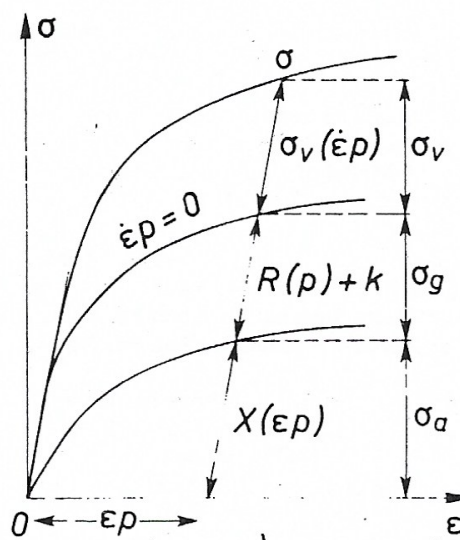

a)

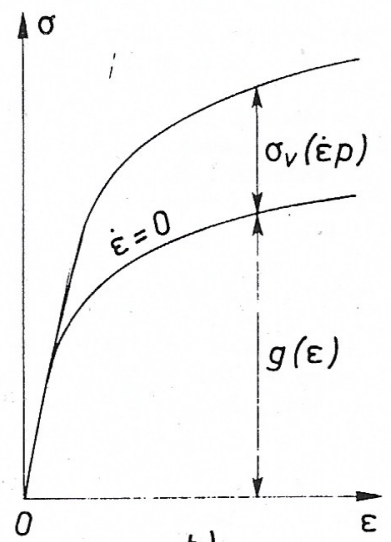

b)
Fig. 3 Stress decompositions in the tensile test

(a) Nonlinear kinematic rule and I.M.G. model [45, 49]

(b) Krempl-Cernocky decomposition $[53,54]$

as in (6) and where the "viscous" stress $\sigma_{v}$ is a power function of plastic strain rate

$$
\sigma_{v}\left(\dot{\epsilon}_{p}\right)=K \dot{\epsilon}_{p}^{1 / n}
$$

This decomposition is illustrated in Fig. 3(a), which shows that curve $\chi+R+k$ corresponds to an equilibrium behavior (zero strain rate).

Let us point out some similarities between such viscoplastic equations, where hardening is described in terms of internal variables and other approaches, using total strain concept and no elastic domain. Such theories $[43,44,45,46]$ are developed in some intermediate range between the above approaches and works by Valanis [47], and use the complete history of observable variables to define the present state. In fact, only some particular events are memorized, when plastic flow reverses [48] or when work dissipation rate becomes negative [49]. If loading reverses, these approaches use instantaneous changes of the characteristic functions, which leads to several difficulties, especially under multiaxial and random loading (as in the Eisenberg's formulation [50] for time-independent plasticity and in the ORNL model [51]). Let us note the recent publication [52] around the three-dimensional generalization of ORNL model for any kind of loading, where updating procedure is not needed, but instantaneous changes still intervene.

The theory by Krempl and his co-workers [53, 54] uses in the one-dimensional case

$$
\dot{\epsilon}=\frac{\dot{\sigma}}{E}+\frac{\sigma-g(\epsilon)}{E k(\sigma-g(\epsilon))}
$$

where $g(\epsilon)$ plays the role of an equilibrium stress-strain curve (zero strain rate) and $k($.$) is the viscosity function. Using the$ notation

$$
F(\mu)=\frac{\mu}{E k(\mu)}
$$

one can write equation (28) as

$$
\sigma=g(\epsilon)+F^{-1}\left(\dot{\epsilon}-\frac{\dot{\sigma}}{E}\right)
$$

$F^{-1}$ plays the role of the aforementioned viscous stress and $g$ ( $\epsilon$ ) represents the sum $X\left(\epsilon_{p}\right)+R(p)+k$ in equation (26); the only differences are the total strain instead of plastic strain and the specific choices of functions $g$ or $k$ in regard to functions $X$ and $\sigma_{v}$. Let us point out the greater flexibility of (26) under cyclic loading because of the difference between actual plastic strain and cumulated plastic strain (that is important to describe separately monotonic and cyclic hardening).

Such a similarity had been advocated by the authors [44], as well as for the three-dimensional formulations. The general properties of the two kinds of modelization are then similar and reproduce many experimental results, especially under varying strain rate or stress rate [54].

Two additional differences can be selected:

1 Under cyclic loading, Krempl intends to use updating procedures; our present developments sustain the idea that such updating is not really necessary, which can greatly simplify the calculation procedures.

2 To describe aging process, time can be directly incorporated into the characteristic functions of the model [53]; we prefer to introduce its effect through additional terms in the evolution equations as mentioned in the foregoing for a recovery effect or by means of additional internal variables $[9,55]$ (see also Part II of the present paper).

The model developed by Guelin, et al. $[45,49]$ uses a similar decomposition of stress

$$
\sigma=\sigma_{a}+\sigma_{g}+\sigma_{v}
$$

where $\sigma_{a}$ is the pure hysteresis and is controlled by an equation quite similar to the nonlinear kinematic rule (5), $\sigma_{g}$ describes the strain hardening, $\sigma_{v}$ is the viscous stress, depending on strain rate through an integral formulation. The decomposition (30) is identical to (26), as shown in Fig. 3: $\sigma_{a}$ corresponds to $\chi, \sigma_{g}$ corresponds to $R+k$. Here also, the differences intervene in the choice of the functions, and under cyclic loading, where updating of some characteristics are needed, especially the stress and the $\sigma_{a}$ variable. With the same global properties, the nonlinear kinematic rule does not need any similar updating; it uses a completely defined set of explicit equations for the internal variable evolutions, for'any kind of cyclic multiaxial (and random) loading.

\section{Conclusion}

The plastic and viscoplastic constitutive equations are under constant development, giving rise to better descriptive possibilities through different formulations.

1 Concerning the classical or less classical plastic and viscoplastic flow theories, one point has been underlined: many approaches considered as different in their general statements and their point of departure lead in fact to similar descriptive possibilities and in some occasions to quite identical flow rules; for monotonic loadings, only small changes in the characteristic functions lead from a typical approach to another one. So the differences seem to be more a question of presentation:

(i) from a set of surfaces,

(ii) from explicit differential equations for a set of internal variables,

(iii) with no internal variables and no yield surface concept.

2 The theories based on internal variables (ii), are in a median situation in regards to the others and it is the opinion of the authors that they give a larger range of applicability with the following approach:

- try the simplest model with a few internal variables;

- if necessary change the characteristic functions or add some complementary effects (time recovery for example) on the basis of physical observations;

- if a particular additional process is evident from experiments, try to introduce it through additional internal variables, obeying particular rules (strain memory effects [31] and history of temperature [9] are such examples).

3 Another point has been pointed out several times in this 
paper, concerning cyclic flow rules with or without updating of variables; our opinion is that updating is not necessary if sufficiently good equations are used for internal variable evolutions (as demonstrated by the so-called nonlinear kinematic rule). The updating procedures are generally involved to transform good monotonic flow rules into not too bad cyclic flow rules. In fact, we have to think in terms of cyclic loadings, even for monotonic ones. These remarks constitute partial opinions, but the authors admit the possibility of good developments of updating procedures if they are written correctly for any three-dimensional nonproportional and random loading.

\section{References}

1 Mandel, J., "Plasticité Classique et Viscoplasticité," Cours du C.I.S.M., Udine, 1971.

2 Halphen, B., and Nguyen, Q. S., "Sur les Matériaux Standard Généralisés," Journale de Mécanique, Vol. 14, No. 1, 1975, pp. 39-63.

3 Sidoroff, F., "On the Formulation of Plasticity and Viscoplasticity with Internal Variables," Arch. Mech., Poland, Vol. 27, No. 5-6, 1975, pp. $807-819$.

4 Chaboche, J. L., "Description Thermodynamique et Phénomènologique de la Viscoplasticité Cyclique avec Endommagement," Thèse de Doctorat, Paris VI, 1978.

5 Cailletaud, G., "Modélisation Mécanique d'Instabilités Microstructurales en Viscoplasticité Cyclique à Température Variable,". Thèse Paris VI, 1979.

6 Armstrong, P. J., and Frederick, C. O., "A Mathematical Representation of the Multiaxial Bauschinger Effect," C.E.G.B. Report RD/B/N 731, 1966.

7 Chaboche, J. L., "Viscoplastic Constitutive Equations for the Description of Cyclic and Anisotropic Behaviour of Metals," Bull. de l'Acad. Polonaise des Sciences, Série Sc. et Techn., Vol. 25, No. 1, 1977, pp. 33-42.

8 Marquis, D., "Etude Theorique et Verification Experimentale d'un Modele de Plasticite Cyclique," Thèse Paris VI, 1979.

9 Cailletaud, G., and Chaboche, J. L., "Macroscopic Description of the Microstructural Changes Induced by Varying Temperature. Example of IN 100 Behaviour,"' ICM3, Cambridge, 1979.

10 Besseling, J. F., "A Theory of Elastic, Plastic and Creep Deformation of an Initially Isotropic Material Showing Anisotropic Strain-hardening, Creep Recovery and Secondary Creep," ASME Journal of Applied Mechanics, 1958, pp. 529-536.

11 Meijers, P., "Description of Multiaxial Creep Behaviour Using the Overlay Model," a short presentation, IUTAM Symp. on Creep in Structure, Leicester, 1980.

12 Miller, A., "An Inelastic Constitutive Model for Monotonic Cyclic and Creep Deformation," ASME Journal of Engineering Materials and Technology, Vol. 98, 1976, pp. 97-105 and 106-113.

13 Rice, J. R., "On the Structure of Stress-strain Relations for Time Dependent Plastic Deformation in Metals," ASME Journal of Applied Mechanics, Vol. 37, 1970, pp. 728-737.

14 Prager, W., "Recent Developments in the Mathematical Theory of Plasticity," Journal of Applied Physics, Vol. 20, No. 3, 1949, pp. 235-241.

15 Ziegler, H., "A Modification of Prager's Hardening Rule," Quarterly of Applied Mathematics, Vol. 17, No. 1, 1959, pp. 55-56.

16 Mroz, Z., Shrivastava, H. P., and Dubey, R. N.; "A Non-Linear Hardening Model and its Application to Cyclic Loading," Acta Mechanica, Vol. 25, 1976, pp. 51-61.

17 Lee D., and Zaverl, F., Jr., "A Description of History Dependent Plastic Flow Anisotropic Metals," ASME Journal of Engineering Materials and Technology, Vol. 101, 1979, pp. 59-67.

18 Edelman, F., and Drucker, D. C., "Some Extensions of Elementary Plasticity Theory," Journal of Franklin Institute, Vol. 251, No. 6, 1951, pp. $581-605$

19 Baltov, A., and Sawczuk, A., "A Rule of Anisotropic Hardening," Acta Mechanica, Vol. 1, No. 2, 1965, pp. 81-92.

20 Shrivastava, H. P., Mroz, Z., and Dubey, R. N., "Yield Criterion and the Hardening Rule for a Plastic Solic," ZAMM, Vol. 53, No. 10, 1973, pp. $625-633$.

21 Lee, D., and Zaverl, F., Jr., "A Description of Deformation Induced Anisotropy in 304 Stainless Steel," Journal of Nuclear Materials, Vol. 79, 1979 , pp. $426-429$

22 Moreton, D. N., Moffat, D. G., and Parkinson, D. P., University of Liverpool, Report No. A/049/80, 1980.

23 Phillips, A., Liu, C. S., and Justusson, J. W., "An Experimental Investigation of Yield Surfaces at Elevated Temperatures," Acta Mechanica, Vol. 14, 1972, pp. 119-146.

24 Bui, H. D., and Zarka, J., "Fondements Expérimentaux et Modèle pour l'Etude du Comportement Global des Métaux," Sciences et Techniques de l'Armement, ler fascicule, 1973, pp. 45-54.
25 Lee, D., and Zaverl, F., Jr., "Further development of Generalized Constitutive Relations for Metal Deformation," G.E. Report No. 79 CRD 179, 1979.

26 Marquis, D., "Sur un Modèle de Plasticité rendant compte du Comportement Cyclique," Zème Congrès Francais de Mécanique, Nancy, 1979.

27 Dafalias, Y. F., and Popov, E. P., "A Model of Non Linearly Hardening Materials for Complex Loading,"' Acta Mechanica, Vol. 21, No. 3, 1975, pp. 173-192.

28 Krieg R D "A Practical two Surface Plasticity Theory," ASME Journal of Applied Mechanics, Vol. 47, No. E3, 1975, pp. 641-646.

29 Dafalias, Y. F., and Popov, E. P., "Plastic Internal Variables Formalism of Cyclic Plasticity," ASME Journal of Applied Mechanics, Vol. 98, No. 4, 1976 , pp. $645-651$

30 Mroz, Z., "On the Description of Anisotropic Work-hardening," Journal of Mechanical Physics Solids, Vol. 15, No. 3, 1967, pp. 163-175.

31 Chaboche, J. L., "Dang-Van, K., and Cordier, G., "Modelization of the Strain Memory Effect on the Cyclic Hardening of 316 Stainless Steel," SMIRT5, Division L, Berlin, 1979.

32 Dafalias, Y. F., "The Concept and Application of the Bounding Surface in Plasticity Theory," IUTAM Symp. on Physical Non-Linearities in Structural Analysis," Senlis-France, 1980, Springer, pp. 56-63.

33 Dafalias, Y. F., "A Novel Bounding Surface Constitutive Law for the Monotonic and Cyclic Hardening Response of Metals," SMIRT-6, Paper No. L-3/4, Paris, 1981.

34 Lee, D., and Zaverl, F., Jr., "A Generalized Strain Rate Dependent Constitutive Equation for Anisotropic Metals," Acta Metallurgica, Vol. 29, 1978, pp. $1771+1780$.

35 Mermet, A., Delobelle, P., and Oytana, C., "Etude Expérimentale du Fluage à Haute Température en Contraintes Biaxiales," Labo. de Méca. Appliquée, Besancon, Rapport No. A 651 3667, 1980.

36 Chaboche, J. L., Policella, H., and Kaczmarek, H., "Applicability of the SRP Method and Creep-fatigue Damage Approach to the LCHTF Life Prediction of IN-100 Alloy," Aalborg-Denmark, AGARD-CP-243, 1978, pp. 4-1 and 4-20.

37 Orowan, E., "The Creep of Metals," Journal of West Scotland Iron Steel Institute, Vol. 54, 1946, pp. 45-53.

38 Lagneborg, R., "A Modified Recovery-creep Model and its Evaluation," Metallurgical Science Journal, Vol. 6, 1972, pp. 127-133.

39 Merzer, A., and Bodner, S. R., "Analytical Formulation of a Rate and Temperature Dependent Stress-strain Relation," ASME Journal of Engineering Materials and Technology, Vol. 101, 1979, pp. 254-257.

40 Malinin, N. N., and Khadjinsky, G. M., "Theory of Creep with Anisotropic Hardening," International Journal Mechanical Science, Vol. 14, 1972, pp. 235-246.

41 Ponter, A. R. S., and Leckie, F. A., "Constitutive Relationships for the Time Dependent Deformation of Metals," University of Leicester, Report 73-6. 42 Harrison, G. F., and Evans, W. J., "A Creep Deformation Map for Nimonic 90-Its Construction, Interpretation and Implications for Life Prediction," International Conference on Engineering Aspects of Creep, Sheffiels, 1980.

43 Krempl, E., "On the Interaction of Rate and History Dependence in Structural Metals," Acta Mechanica, Vol. 22, 1975, pp. 53-90.

44 Cernocky, E. P., and Krempl, E., "A Theory of Viscoplasticity Based on Infinitesimal Total Strain," Acta Mechanica, Vol. 36, 1980, pp. 263-289.

45 Guelin, P and Stutz, P. "Une Nouvelle Classe de Lois de Comportement Décrivant les Grandes Déformations Visco-Elastoplastiques,' Arch. Mech. Stosow, Vol. 29, No. 1, 1977.

46 Stouffer, D. C., and Bodner, S. R., "A Constitutive Model for the Deformation Induced Anisotropic Plastic Flow of Metals," International Journal of Engineering Science, Vol. 17, 1979, pp. 757-764.

47 Valanis, K. C., "A Theory of Thermoviscoplasticity without a Yield Surface,"'Arch. Mech. Stosow., Vol. 23, No. 4, 1971, pp. 517-533.

48 Cernocky, E. P., and Krempl, E., "A Theory of Thermoviscoplasticity based on Infinitesimal Total Strain," International Journal of Solids Structures, Vol. 16, 1980, pp. 723-741.

49 Terriez, J. M., "Sur un Schéma à Mémoire Discrète du Comportement des Continus Irréversibles," Thèse Doctorat, University Grenoble, 1980.

50 Eisenberg, M. A., "A Generalization of Plastic Flow Theory with Application to Cyclic Hardening and Softening Phenomena," ASME Journal of Engineering Materials and Technology, 1976, pp. 221-228.

51 Pugh, C. E., "Constitutive Equations for Creep Analysis of LMFBR Components-Advances in Design for Elevated Temperature Environment,' ASME Publication, G-00092, 1975.

52 Murakami, S., and Ohno, N., "A Constitutive Equation of Creep for High Temperature Component Analysis: an Alternative Proposition to ORNL Constitutive Equation of Creep," SMIRT-6, Paris, Paper L 7/3, 1981.

$53 \mathrm{Krempl}$, E., "Viscoplasticity Based on Total Strain. The Modelling of Creep with Special Considerations of Initial Strain and Ageing," ASME Journal of Engineering Materials and Technology, Vol. 101, 1979, pp. $380-386$

54 Liu, M. C. M., and Krempl, E., "An Uniaxial Viscoplastic Model Based on Total Strain and Overstress," Journal of Mechanical Physics Solids, Vol. 27, 1979, pp. 377-391.

55 Cailletaud, G., Culie, J. P., and Kaczmarek, H., "Mechanical Description of Viscoplastic and Damage Behaviour in Presence of Microstructural Instabilities Induced by Variable Temperature," 3rd IUTAM Symposium on Creep in Structures, Leicester, 1980. 